\title{
Review on Current Status of Vaccines against Parasitic Diseases of Animals
}

\author{
Bedaso Kebede $^{1^{*}}$, Teshale Sori ${ }^{2}$ and Bersissa Kumssa ${ }^{2}$ \\ ${ }^{1}$ Veterinary Drug and Animal Feed Administration and Control Authority, Ministry of Livestock and Fisheries Development, Addis Ababa, Ethiopia \\ ${ }^{2}$ College of Veterinary Medicine and Agriculture, Addis Ababa University, Addis Ababa, Ethiopia
}

"Corresponding author: Bedaso Kebede, Veterinary Drug and Animal Feed Administration and Control Authority, Ministry of Livestock and Fisheries Development, Addis Ababa, Ethiopia, Tel: 0913136824; E-mail: Kebede.bedaso@yahoo.com

Rec date: Feb 15, 2016; Acc date: Apr 04, 2016; Pub date: Apr 06, 2016

Copyright: ( 2016 Kebede B, et al. This is an open-access article distributed under the terms of the Creative Commons Attribution License, which permits unrestricted use, distribution, and reproduction in any medium, provided the original author and source are credited.

\begin{abstract}
Parasitic diseases are one of the most common problems that confront the health and productivity of animals worldwide. Parasites are responsible for organ condemnation, zoonoses and huge economic losses in animal production. Various control methods have been implemented to minimize or curb losses caused by parasitic diseases. Chemotherapy and chemoprophylaxis are the most widely used control approaches all over the world. However, development of drug resistance, high price of the drugs, unavailability and growing concern about drug residues hinder the success of this approach. Vaccination is regarded as one of the best alternative method for the control of parasites in the future. In an attempt of to develop commercial vaccines against economically important parasites researchers have so far focused on identifying target antigens. Some of these include ticks salivary gland antigens, secretory and excretory antigens of helminthes and hidden antigens of Heamonchus contortus. As a result of this effort several candidate antigens have been identified, vaccines prepared from them and tested for their suitability and efficacy. However, most of these vaccines have not been widely utilized. Information about the regulations and standard operating procedures that apply to licensing of production and marketing of parasite vaccines is scanty. Therefore, the objective of this article is to review the current status of vaccines against parasitic diseases of animals.
\end{abstract}

Keywords: Parasites; Vaccines; Control

\section{Abbreviations}

IgM: Immunoglobulin M; IgG: Immunoglobulin G; IgA: Immunoglobulin A; IgE: Immunoglobulin E; IgD: Immunoglobulin D

\section{Introduction}

Parasitic diseases are global problems and considered as a major obstacles in curtailing health and lowering productivity of animals. Both endoparasites that live inside the body and ectoparasitesthat live on the body like ticks, mites, lice, fleas and flies attack the body surface which is responsible for huge economic losses in animal production. After several times of exposure to nematode infection cattle and sheep gradually become resistant to reinfection especially when they become adult animals. This fact provides the basis for believe and search of vaccines against parasitic diseases. However, the development of immunity against parasites depends on numerous factors including breed, genetic makeup of individuals, age and several characteristics of co-infecting nematode species. In addition, management factors such as provision of clean pasture and adequate nutrition can greatly influences attempts to control infection. This brief list could be extended and all of these factors need to be considered when devising vaccination strategies. Vaccine development against parasites faces several fundamental challenges like the isolation of native antigens from none blood feeders which elicit protective immunity if delivered to the immune system in an appropriate manner [1,2].

Helminth parasites have had a significant impact on animal health and/or productivity from the time that animals were first domesticated and then either farmed intensively or kept as companions by humans. Parasites of domestic animals have been controlled using several antiparasitic drugs worldwide. But the use of these antiparasites will not continue in the foreseeable future. This is due to insurgence of resistance against the drugs by the parasites of animals, high cost of drugs, less availability and food safety issues concerning drug residues. These limitations of antiparasitic drugs as seen in many regions of the world provide a window of opportunity for the development and use of alternative control strategies. Recent research findings have shown that it is possible to stimulate protective immunity in animal hosts against parasites by vaccination [3]. Vaccines with the ability to interrupt the life cycle of certain Helminth parasites have been used for effective control of helminthosis in herd or flock of animals [4]. Similarly, effective vaccines have also been developed and their efficiency has been tested against other parasitic diseases.

Vaccination could be applied either to protect the most susceptible animals in a flock/ herd or to minimize the buildup of larvae on pasture and so reduce the rate of infection in susceptible animals. However, vaccines have not been widely used at field level to control the widely distributed parasitic infections globally. Even though several vaccines have been developed and their efficacy published, they have not been commercialized due to a number of technical factors [5]. The advancement of molecular techniques is also considered as a favorable condition for development of new vaccines in the near future. Reliable and up to date scientific information is crucial for understanding and application of vaccines against parasitic diseases.

Therefore, the objective of this article is to review the current status of vaccines against parasitic diseases of animals. 


\section{Pathogenic Significance of Parasites}

Parasitic diseases of animals have been known to cause a wide range of pathologic conditions in their hosts. For example the developmental stages of gastric parasites cause a reduction in the functional gastric gland mass. This has been shown to be responsible for the production of non-acidic gastric juice due to replacement of parietal cells by rapidly dividing; undifferentiated, non-acid-secreting cells [6]. The ultimate outcome is reduced feed utilization and loss of weight.

Some parasites suck large volumes of blood and cause clinical aneamia are Heamonchus contortus, Anclyostoma species and etc. Their hematophagous nature seepage of blood from damaged mucosa and degeneration of epithelial cells of gastrointestinal tract has been responsible for greater morbidity and mortality of animals. For instance blood loss as a result of Heamonchus, Fasciola, other intestinal helminthes and protozoa species have been known to cause such clinical episodes. Some parasites have also been known to cause organ damage due to either mechanical effect or inflammatory reactions are Fasciola, Schistosoma, Lungworm, Ascarid, Flaroids and etc. This will cause severe morbid processes and reduction in productive and reproductive performances. There are also parasitic organisms that cause systemic illness such as Trypanosomosis, Toxoplasmosis and Babesiosis [6].

Strongylus species especially Strongylus vulgaris damage cranial mesenteric artery and its main branches. This artery forms thrombus due to larval damage endothelium together with the marked inflammation and thickening of the arterial wall. Finally, equine colic emerges particularly in foals [6].

Ticks are known to cause skin damage which result in deterioration of the market value of skin, tick paralysis, anemia which leads to death and transmission of infections. Mites, fleas and lice cause skin damage due to delayed type hypersensitivity reactions; myasis producing insects cause annoyance and death [7].

In general, parasitic diseases play great role in the socio-economic values and public health significances.

\section{Control of Parasites}

\section{Chemotherapy and chemoprophylaxis}

Chemotherapy as a control approach against parasites has long been used in all parts of the world. These have been widely applied through tactical and/ or strategic methods but are not without risks. A parasiticide is a poison that is more toxic to parasites than to their hosts. The degree of discrimination is sometimes small, sometimes considerable, but never perfect, so that application of parasiticides always entails some hazard to the host. As a matter of fact it is always easier to explain the deleterious effects that parasiticides quite frequently exert on the host than to explain how they kill parasites. For instance organophosphates and carbamates inhibit cholinesterase causing accumulation of acetylcholine and blockage of the respiratory muscles followed by death due to cerebral anoxia [8]. Nowadays, Chemotherapy and Chemoprophylaxis are not promising. This is due to development and escalating issue of drug resistance, food safety concern arising from residues and environmental issues. Drug resistance can be defined as the loss of the initial sensitivity of parasites to the effect of substances to which they were previously sensitive. For example, there are several reports on resistance of ivermectin including resistance in Cooperia species in several countries in the southern hemisphere [9].

\section{Integrated control}

The term integrated pest control implies a rational use of a combination of biological, bio-technological and chemical control measures in all farming practices or breeding strategies in order to reduce the use of chemical control agents to an absolute minimum. A classic example of this approach is the combination of grazing management and anthelmintics treatment [10]. The relevance of an integrated approach has grown for several reasons. A single antiparasitic treatment of animals in an infected environment proved to have a very transitory effect as it becomes reinfected shortly after treatment. The sparing use of parasiticides has been advocated due to increasing problems with chemical resistance. A combination of two or more effective methods may substantially reduce infection levels and give appropriate control. Lastly, under some conditions control programs of very high efficacy for example use of sustained release devices may be unwanted as it may compromise the development of immunity. For these reasons an integrated approach combining different methods is likely to achieve the best control [10].

\section{Biological control}

Biological control may be defined as the use of one living organism introduced into the environment to obtain control of a target parasite and thereby reducing the population growth of the latter below a threshold where it causes minimal clinical problems and /or economic losses. For instance, nematophagous fungi were shown to reduce populations of preparasitic stages of nematodes significantly. These fungi are relatively easy to culture and can be released into the environment of the target organisms in a controlled fashion. The fungus Duddingtonia flagransis is very efficient in controlling most of the economically important gastrointestinal nematodes of grazing livestock by reducing pasture infectivity [11].

\section{Immunoprophylaxis}

Immunization is the most desirable means of combating infectious agents. The fact that vaccines are potentially safer, cheaper and more efficacious as prophylactics than drugs is based mainly on our experience to date with antimicrobial vaccines. Exceptionally vaccine used against bovine lung worm infection that is prepared from irradiated larvae of Dictyocaulus viviparous there are no commercially available vaccines for the control of most of the parasitic infections of animals $[11,12]$. Diseases caused by parasites are prevalent globally and account for enormous losses of livestock productivity and zoonoses.

\section{Vaccine Development against Parasites}

Few vaccines against parasites have been developed and reached the market $[7,13,14]$. Although some of the arguments that explain market failure are correct in their own right there are many more factors that affect commercial success [15]. Some of the most important factors are summarized below:

\section{Quality}

A special situation is the production of vaccines from feaces of live animals for example coccidiosis vaccine. The quality assurance system comprises the microbiological status of these animals and preferably 
Page 3 of 8

specific pathogen free animals must be used. Additionally a validated final product test on sterility or purity is required [16].

\section{Safety}

Clearly the product must be safe to the target animal but it must also be documented not to pose a danger to other animals or man that may come into contact with the product or to the environment. In addition, the safety of an overdose or repeated doses of the vaccine must be shown. A special requirement is that live vaccine strains must be stable that means should not revert to virulence during consecutive passages. In general, a vaccine has to be produced with a limited number of passages from the master seed stock which is usually limited to five passages. The safety of the parasite vaccines at the lowest and highest passages should be shown in animal studies using the most sensitive target animal species. This is a mandatory prerequisite before marketing the product [17].

\section{Efficacy}

A product must be able to do what is claimed. For instance, limit parasite multiplication or the development of clinical signs. This need to be shown in large number of target hosts under various geographical locations and different animal farming systems [18].

\section{Potency}

The manufacturer must provide data that guarantees the efficacy of a product over the entire shelf life. This is a major hurdle in vaccine development. The discovery of the protective effect of specific immunogenparasite strain or partially purified parasite fraction is usually done or further established by vaccination challenge experiments. Such experiments are preferably not used as potency tests as they involve animals for experimentation and take long time in case of some live vaccines this is a serious problem. An alternative test must be developed and potency test carried out at the time of batch release which has predictive value as to the efficacy of that particular batch at the end of shelf life. Hence, the dynamics of the signal of the potency test must be studied over the period of the shelf life and correlate with the level of efficacy (real time stability data). If the protective mechanism of immunity against a specific parasite is not known it may take years before an accurate potency test is developed. This has been one of the standing problems in commercializing parasitic vaccines [19].

\section{Consistency of production}

Clearly from the legislation in certain countries like USA a license must be obtained for each production facility where the vaccine is being produced in addition to a license to sell the product at a certain market. Data must be presented to show that at least three consecutively produced batches of product meet the quality requirements safety, purity and potency specified for that product. Thus, for most of the vaccines produced against parasites and whose usefulness has been published do not clearly show consistency of production [20].

\section{Product profile}

Product profile is a description of the composition of the product, the target animal to be vaccinated (species, minimal age, etc), the specific safety and efficacy claims, the route and method of administration, shelf life and physical presentation. A multitude of approaches are used to discover parasite strains or fractions there of that induce protection in the host [12]. One should realize that this is only the first step in vaccine development and the critical component must induce a significant level of protection. The next step is to formulate specific safety and efficacy claims that add to the product profile. These claims can be clarified by experimentation on model animals but should necessarily be conducted on target animals. Clearly, it must be specified for which animal species the product is intended. In addition, the category must be stated for example minimal age of the target animal, whether the product can be used safely in pregnant animals or is intended for specific use as vaccine for broiler chickens but opposed to breeder chickens or layer flocks. An important factor may be the presence of maternal immunity in young animals. This should possibly affect the induction and on set of vaccine induced immunity then this must be studied. Results may be prompt to the manufacturer to recommend not vaccinating animals below a certain age. Most products so far published as effective vaccines do not show these profiles [18].

\section{Onset and duration of immunity}

Any of the claims regarding onset and duration of immunity following single vaccination must be documented either supported by existing literature or by experimental data. It should also include the specific time for booster vaccination and such data should emanate from challenge studies involving laboratory animal models and target animals. The results of such tests usually take longer than two years to complete [21].

\section{Compatibility}

When a vaccine claimed to be physically mixed with another product and subsequently administered the data of safety and efficacy studies must be presented to support such simultaneous use [22].

\section{Routes of administration}

Different vaccines are administrated through different routes as oral, intradermal, subcutaneous and intramuscular or by topical application. For each routes of administration safety and efficacy data must be available and this must be clearly stated in the dossier and on the leaflet [23].

\section{Immunity against Parasite Antigens}

\section{Immunity to protozoa}

Most protozoans are fully antigenic but due to their adaptation to a parasitic existence they have developed mechanisms through which they may survive in the presence of an immune response. Therefore, like other antigenic particles parasitic protozoa can stimulate both humoral and cell mediated immune responses. In general, antibodies serve to control the stages of protozoa that exist free in the blood stream and tissue fluids whereas cell mediated immune response are directed largely against intracellular forms. Serum antibodies directed against parasitic protozoa surface antigens may opsonize, agglutinate or immobilize them. Antibodies together with complement and cytotoxic cells may kill them and some antibodies called ablastins may act to inhibit parasitic protozoan enzymes in such a way that their replication or metabolism is prevented [24]. 


\section{Immunity to helminths}

Antibodies like the IgM, IgG and IgA isotypes are produced in response to helminth antigens an increasing body of evidence suggests that the most significant immunoglobulin isotype involved in resistance to helminth is IgE. As a result, the development of a worm burden provokes a local acute type I hypersensitivity reaction in the parasitized regions of the body. The combination of helminth antigens with mast cell bound IgE leads to mast cell degranulation and release of vasoactive amines. These compounds stimulate smooth muscle contraction and increase vascular permeability which results in dislodgement and expulsion of worms [25]. There has been evidenced to suggest that sensitized T-lymphocytes in the intestinal mucosa to be involved in resistance against helminths such as Trichinellaspiralis and Trichostrongyluscolubriformis infections. Sensitized T-lymphocytes depress the activities of helminths by two mechanisms. First, the developments of an inflammatory response of the delayed hypersensitivity type tend to attract mononuclear cells to the site of larval invasion and render the local environment unsuitable for growth or migration. Second, cytotoxic lymphocytes may be capable of causing larval destruction [26].

\section{Immunity to arthropods}

When arthropods bite an animal they inject saliva. Their saliva contains antigens and therefore induces immune response. Some salivary components are of low molecular weight and as a result they cannot function as normal antigens. They may however bind to skin proteins such as collagen and then function as haptens stimulating a cell mediated response. On subsequent exposure these haptens will induce a delayed hypersensitivity reaction. Other salivary antigens may bind to epidermal largerhan's cells and induce cutaneous basophil hypersensitivity reaction associated with IgG antibodies and a basophil infiltration. The third type of response to arthropod saliva is an IgE response with an associated type Ihypersensitivity. This response may induce a severe inflammatory response in the skin and as a result cause severe discomfort to the bitten animal. Each of these three types of response may modify the skin in such a way that the feeding of the offending arthropod is impaired and the animal may therefore be a less attractive source of food [27].

In general, for all classes of parasites protozoa, helminths and arthropods the mechanism of natural immunity has been understood. The knowledge of immunity to natural infection forms a benchmark for development and production of efficacious vaccines to induce protective immunity. Hence, a number of vaccines have so far been developed and tested [28].

\section{Vaccination against Parasitic Infection}

Successful vaccination against protozoan infections of domestic animals is currently limited to Babesiosis, Toxoplasmosis, and Theileriosis. Animals that recover from acute Babesiosis are resistant to further clinical disease and this immunity has been considered to be a form of premunity. It is possible to infect young calves deliberately so that they will acquire infection while they are still relatively insusceptible to disease and later become resistant to re-infection. Many attempts have been made to immunize poultry against coccidian but have been clear that only infection with viable organisms can induce protective immunity. Thus repeated dosing with small number of oocysts can provide some protection. Unfortunately, this technique may provoke severe reactions that require treatment with coccidiostats.
Alternatively oocysts may be attenuated by ionizing radiation. In case of well-adapted organism such as Toxoplasma gondii not only does infection rarely lead to disease but it also results in the development of strong lifelong immunity to reinfection. Because of this it would be difficult to produce vaccine against this organism that would improve significantly on the natural infection. It may be desirable to develop a vaccine for cats which could inhibit oocyst production and thus break that segment of transmission cycle [24].

It is not surprising that considering the ineffectiveness of the host response to helminths that vaccines are not widely available. Since vaccines consisting of dead helminths or their extracts have been uniformly unsuccessful in conferring protection because of this studies have tended to concentrate on the use of irradiated larvae. Experimentally it has been shown that irradiated metacercariae can reduce Fasciolahepatica burden in calves. Whereas irradiated ova of Ascaridiagalli protected chickens against challenge by this helminth and Ultraviolet-irradiated Ascarissuum eggs can confer protection in pigs. However, very few of these preparations have been commercially produced with success. Perhaps the most important of those vaccines that have been widely used to protect livestock is the vaccine against verminous pneumonia caused by the lungworm Dictyocaulusviviparous. A similar type of vaccine has been used to protect puppies against the hookworm Ancylostomacaninum. In this case, irradiated larvae are administered three days after birth in order to provide immunity [29].

The important antigens of most arthropod parasites are found in the saliva. Althoughvaccination against salivary antigens is unlikely to be very effective in conferring effective immunity against blood feeding arthropods, there is an alternative approach. Since many of the arthropods of veterinary importance take the blood of their host into their digestive tract it follows that they will also take up immunoglobulins, complement components and cells. This suggests that if an animal were immunized with internal antigens from the parasite this could lead to local damage. The internal antigens have been called hidden or concealed antigens since under normal circumstances the host would not encounter those [30].

The mange mite Demodex folliculorum appears to be a normal symbiont commonly present in hair follicles and only occasionally cause disease. Animals suffering from generalized demodicosis have normal neutrophil function and respond normally to vaccines or other foreign proteins. Biting fleas secrete saliva into the skin wound. Experimental vaccines containing the major antigens from the cat flea midgut have been able to reduce flea population on dogs and the female fleas recovered from these immunized animals produced significantly fewer eggs. This suggests that vaccination may eventually be a method of controlling flea populations [31]. It has been suggested that local cell mediated and immune complex hypersensitivity to tick saliva may restrict the blood flow to the tick reduce its food supply and stunt its growth. It has been possible to immunize guinea pigs with tick homogenates and show that ticks feeding on these animals have reduced fertility and egg production. Vaccines made against antigens from the intestine of the tick Boophilus microplus was shown to inhibit tick production. Recombination tick vaccine based on such antigens Bm86 is available in Australia [32]. On the other hand vaccines containing salivary antigens may be more effective in reducing tick feeding and thus the transmission of pathogens. The antibodies produced inhibit endocytosis by gut endothelial cells and prevent the tick from engorging fully. In addition, tick feeding on vaccinated animal produce significantly fewer eggs than normal [28]. The larvae 
Page 5 of 8

of the warble flies Hypoderma bovis and Hypoderma lincatum migrate through body tissues. Vaccination with cloned hypoderma proteins has effectively protected animals against subsequent infections [24].

\section{Types of Vaccine}

\section{Live vaccines}

Live vaccines have shorter shelf life and less stable vaccines prepared either from the etiologic agents or less pathogenic strains. They have risks associated with possible spread of the parasites to other susceptible animals. In this respect one can distinguish two groups of live vaccines those that induce self-limiting infections and those that result in chronic infections. Vaccines that cause self-limiting infections have been based on the use of parasite strains that cause infections which does not usually present. They have no risk to the environment since the life cycle of the parasite is not perpetuated. Requirements are restricted to providing evidence that the biological characteristics of the vaccine strains do not change upon consecutive passage. This is referred to as reversion to virulence even in cases that a wild type strain is used for vaccination. Examples of the simplest form of such vaccines are the live vaccines against coccidiosis in chickens [33].

The virulence of parasite strains derived from a single isolate can be variable. For example, using Babesiabovis isolatespassage through splenectomised animals can select for strains of reduced virulence. Such parasite strains are being used to vaccinate cattle in Africa and Australia. The infection develops less virulently and the animals develop immunity against subsequent challenge infection [34]. Attenuation can also be brought about by repeated passage in vitro which is the basic technique used to select for the vaccine strains of Theileriaannulata that are used for example in India and Israel [35].

Many parasite species have complicated life cycles characterized by distinct life cycle stages sometimes involving more than one host. In cases where the early life cycle stages are sufficiently immunogenic to induce protective immunity selection for parasite strains with truncated life cycles is another strategy to develop vaccines. A major advantage is that spreading of the vaccine strain in the environmental does not occur. An example is the Toxoplasma gondii S48 that is used in a vaccine against abortion in sheep due to primary Toxoplasma gondii infection during pregnancy. This strain has the capacity to develop from the tachyzoite into bradyzoite stage but does not form tissue cysts. The tachyzoites induce a transient infection in the host while triggering protective immune reactions [36]. Irradiation of parasites has also been used as a mechanism to truncate the life cycle. The live vaccine against lungworm infection in cattle contains L3 larvae of Dictyocaulus viviparus that do not develop further than the L4 stage. Vaccinated cattle are immune to challenge infections with L3 larvae [37].

Live vaccines can be also being developed from parasites that cause chronic infections. In this case the parasites show a tendency to survive in the host for longer periods of time in which case chemotherapeutics cure of the infection is required. An example of this type of live vaccine is vaccine developed against Theileria parva infection [38]. This vaccine is based on isolates of virulent Theileria parva strains which are used to infect cattle that are simultaneously treated with long acting tetracycline preparations to control infection.

\section{Killed vaccines}

Killed vaccines by themselves usually do not induce protective immunity and an appropriate adjuvant and formulation must be developed. In these cases special attention must be given to the safety of the adjuvant used [39]. Aluminum salts water in oil and oil in water and saponins are commonly used as adjuvants [40]. Killed vaccines are more stable and have longer shelf life. Killed vaccines could be prepared from whole organisms or from their parts or products. If no live vaccine strains are available or the use of live vaccines is undesirable one may want to inactivate the parasites prior to the formulation of a vaccine. Examples of such vaccines are the vaccine against abortion in cattle due to Neospora caninum infection [39] and a vaccine against giardiasis in dogs [41]. The major issue with these vaccines is the efficiency of inactivation and experimental evidences must be provided that supports the efficacy of inactivation. Vaccines against Babesiosis and Theileriosis have been developed successfully but both depend up on the use of attenuated organisms [42]. Similarly inactivated whole organism vaccine against schistosomiasis has been formulated. Vaccination with irradiation attenuated larvae has been shown to be effective in rodents and primate models and has also been used in large scale field trails against infection with Schistosoma bovis in Sudan [43]. The most promising of these are the 9P28 KDa antigens known to have glutathione-S-transferase activity which have given very promising results in infections involving Schistosoma mansoni in rodents and primate models [44].

Vaccine development against larvae of cestodes has also got some momentum. Larval stages Taeniaovis and Taeniahydatigena in sheep are an important economic problem due to condemned infected carcasses [45]. The early success of the irradiated vaccine used against lungworm raised hopes that similar vaccines would be effective against the Trichostrongyle species responsible for gastrointestinal infections in cattle and sheep. A number of programs are now using defined and recombinant antigens as the basis of vaccines principally targeted at the important parasites of sheep such as Haemonchus contortus and Trichostrongylus colubriformes. Significant progress has been made using molecules expressed at the surface of the worms intestinal cells [46].

\section{Success and failure of vaccines against parasites}

Successful vaccines have been developed infrequently and have been characterized by use of widely contrasting technologies. A crude and simple vaccine employing whole irradiated nematode larvae developed during 1950's effectively used for the control of lungworms in cattle called Dictyocaulus viviparous [47]. A similar approach has been proved effective for controlling infection with the sheep lungworm called Dictyocaulus filaria [48] and the canine hookworm Ancylostoma caninum [49]. A rewarding advance occurred towards the end of the last century when a vaccine employing a recombinant antigen that conferred protection against a metazoan parasite Taeniaovis was developed. Similar strategies were then employed to develop recombinant vaccines against other cestodes parasites Taeniasaginata and Echinococcusgranulosus [50].

The most recent recombinant or subfraction lungworm vaccines have not achieved levels of efficacy in any way comparable to the crude whole organism vaccine [51]. Despite the efforts of many research groups from different corners of the world the success achieved with recombinant cestodes vaccines has not been shown in the case of nematodes $[1,51,52]$. Similarly there have been a large number of 
unsuccessful attempts to develop vaccines for helminths. Following early experiments where sheep were immunized systemically with ground up worms many research groups around the world have tested a wide variety of worm. Most of these attempts were ineffective in terms of inducing strong protective immunity and some even exacerbated worm infections.

Subunit vaccines have also been tried against some parasites. This was based on more detailed analysis of the immune response acquired after natural infection or vaccine induced immunity that led to the discovery of critical antigenic components of an organism that can be used in a vaccine. An adjuvant is required for the induction of protective immunity. The vaccine against Babesiosis of dogs due to Babesiacanis infection is one such example. It contains soluble antigens secreted or excreted from the parasites and saponin is used as adjuvant [53]. A vaccine against leishmaniosis in dogs based on partially purified fructose-mannose ligand (FML) has been developed commercially and is used as a prophylactic vaccine [54]. A special product is a vaccine against coccidiosis in broilers that is based on the protective effect of vaccination of the breeder hens. In some cases the antigens are produced using recombinant DNA technology. The best example is the vaccine against Taeniaovis in sheep which is based recombinant parasite antigens that induce antibodies that block the attachment of oncospheres to the gut epithelium [18]. Saponinadjuvant was shown to be most efficacious. Another example is the recombinant vaccine produced from gut wall antigens of the cattle tick Boophilusmicroplus [55]. Up on vaccination of cattle high levels of antibodies to the gut wall of ticks are produced. During feeding of the tick on the vaccinated animal these antibodies are ingested and destroy the gut epithelium of the tick thus killing the parasite. Some protein antigens such as the H11 protein from Heamonchus contortusare highly effective in native form but seemingly lose a critical structural feature which accounts for immunogenicity when produced in recombinant form [56].

Several reasons are responsible for the failure of a vaccine to confer protective immunity in animals. In some cases the vaccines may actually be infective because they contain the wrong strain of organisms or the wrong antigens. The method of production may have destroyed the protective epitopes or there may simply be insufficient antigens in the vaccine formulations of much greater significance is the failure of an effective vaccine to stimulate immunity. In some cases this may be attributed to unsatisfactory administration. If animals in incubation period are vaccinated the vaccine may be given too late to affect the course of the disease. More commonly the animal may fail to mount an immune response [57].

The second type of vaccine failure occurs when the normal immune response is suppressed. For example, stress like pregnancy, extremes of cold and heat, fatigue and malnourishment and heavily parasitized may reduce a normal immune response of animals probably because of increased steroid production. This is usually caused by the presence of passively derived maternal immunity in young animals [24]. Many of the failures in vaccine efficacy may be attributable to an inability to conform to one or more of the following requirements. Firstly, antigen presenting cells must be stimulated so that they process antigen efficiently and release appropriate interleukins. Secondly, both $\mathrm{T}$ and $\mathrm{B}$ cells must be stimulated so that they generate large numbers of memory cells. Thirdly, helper and effector T cells must be generated to several epitopes in the vaccines so that variations in major histocompatibility complex (MHC) class II polymorphism and epitopes properties are overcome and finally the antigen must persist in appropriate sites in lymphoid tissues so that antibody producing cells are generated over a period of time and protection persists for a long period $[24,45]$.

\section{Prospects}

Studies have shown that vaccination will be used as one of the effective means for the control of parasitic diseases of animals. However, at present a number of regulatory and pharmaceutical vigilances limit the utility of vaccines. It is anticipated that more harmonization of legislation that will facilitate the production and marketing of commercial products will be realized in the future. Specific requirements will be formulated for parasites vaccines in pharmacopoeias of which the monograph of the live coccidiosis vaccines will be the best and first of its type. With the more advancement of molecular techniques in vaccine development and production new regulations will are formulated in the near future [57].

\section{Conclusion and Recommendations}

The health and productivity of domestic animals is confronted by parasitic diseases caused by helminths and anthropods worldwide. In addition in many cases they also affect human health. Control of parasitic diseases has historically focused on the use of chemotherapy and chemoprophylaxis all over the world. However, this method of control is hindered by the development of drug resistance, high price of drugs, unavailability and growing concern about drug residues. In an attempt to develop commercial vaccines against economically important parasites researchers have so far focused on identifying target antigens. In the near future the use of vaccination against parasitic diseases of animals in veterinary health services is expected to contribute significantly in promoting livestock productivity. Research results showed that several efficacious vaccines have been produced but not yet widely commercialized. This is due to various regulatory and pharmacological vigilances which are often the first requirement by the regulatory and licensing authority. As a result it is hoped that in the future vaccination against parasitic diseases will be used as one of the best alternatives in the control of parasitic infections. Based on the above concluding remarks the following recommendation points are suggested:

Further studies should be conducted to develop vaccines against economically important animal parasites.

Regular standard operating procedures for production and quality control of efficacious vaccines should be established.

Factors that compromise the immune system like malnutrition, heavy parasite burden and stress should be reduced to acceptable level before the use of vaccines.

\section{References}

1. Knox DP, Redmond DL, Newlands GF, Skuce PJ, Pettit D, et al. (2003) The nature and prospects for gut membrane proteins as vaccine candidates for Haemonchus contortus and other ruminant trichostrongyloids. Int J Parasitol 33: 1129-1137.

2. Smith WD, Smith SK, Murray JM, Lidell S, Knox DP (1993) Vaccines against metazoan parasites. Patent Application No. PCT/GB.

3. Knox DP, Redmond DL, Skuce PJ, Newlands GF (2001) The contribution of molecular biology to the development of vaccines against nematode and trematode parasites of domestic ruminants. Vet Parasitol 101: 311-335. 
4. Harrison GBL, Pulford HD, Hein WR, Barber TK, Shaw RJ, et al. (2003) Immune rejection of Trichostrongylus columbriformis in sheep; a possible role for intestinal mucus antibody against an L3 specific antigen. Parasite immunol 25: 45-53.

5. Seifert SH (1996) Tropical Animal Health. CTA, Kluwer Aca. Publishers, The Netherlands, p: 123.

6. Urquhart GM, Armour JL, Duncan AM, Dunn FW, Jennings J (1996) Veterinary Parasitology. 2nd edn. The Faculty of Veterinary Medicine, University of Glasgow, Blackwell, Science, Scotland.

7. Furman DP, Loomis EC (1984) The ticks of California (Acari: ixodid). University of California Publications. Bull Calif Ins Survey 25: 1-239.

8. Georgi Jay R (1980) Parasitology for Veterinarians. 3rd edn. CTA, Kluwer Acad Publishers, The Netherlands, p: 397

9. Michel JF (1969) The epidemiology and control of some nematode infections of grazing animals. Adv Parasitol 7: 211-282.

10. Larsen M (1999) Biological control of helminths. Int J Parasitol 29: 139-146.

11. Emery DL, McClure SJ, Wagland BM (1993) Production of vaccines against gastrointestinal nematodes of livestock. Immunol Cell Biol 71 : 463-472.

12. Cornelissen AW, Schetters TP (1996) Vaccines against protozoal diseases of veterinary importance. FEMS Immunol Med Microbiol 15: 61-72.

13. Dalton JP, Mulcahy G (2001) Parasite vaccines--a reality? Vet Parasitol 98: 149-167.

14. Vercruysse J, Knox DP, Schetters TP, Willadsen P (2004) Veterinary parasitic vaccines: pitfalls and future directions. Trends Parasitol 20: 488-492.

15. Schetters T (1995) Vaccine development from a commercial point of view. Vet Parasitol 57: 267-275.

16. Vermeulen AN, Schaap DC, Schetters TP (2001) Control of coccidiosis in chickens by vaccination. Vet Parasitol 100: 13-20.

17. Sutkowsky EM, Gruber MF (2006) Regulatory consideration in the nonclinical safety assessment of adjuvanted preventive vaccines. In Immunopotentiators in Modern Vaccines. Elsevier Academic Press, London, pp: 343-359.

18. Harrison GB, Shakes TR, Robinson CM, Lawrence SB, Heath DD, et al. (1999) Duration of immunity, efficacy and safety in sheep of a recombinant Taenia ovis vaccine formulated with saponin or selected adjuvants. Vet Immunol Immunopathol 70: 161-172.

19. Stowers A, Carter R (2001) Current developments in malaria transmission-blocking vaccines. Expert Opin Biol Ther 1: 619-628.

20. Rickard MD, Harrison GB, Heath DD, Lightowlers MW (1995) Taenia ovis recombinant vaccine--'quo vadit'. Parasitology 110 Suppl: S5-9.

21. Miller TA (1965) Persistence of immunity following double vaccination of pups with x-irradiated Ancylostoma caninum larvae. J Parasitol 51: 705-711.

22. Miller TA (1978) Industrial development and field use of the canine hookworm vaccine. Adv Parasitol 16: 333-342.

23. Connan RM (1994) Calfhood vaccination for dictyocaulosis. Vet Rec 134: 99.

24. Tizard I (1987) Veterinary Immunology. An introduction. 3rd edn. WB, pp: 234-247.

25. Coyne CP, Brake D (2001) Characterization of Heamonchus contortus derived cell populations propagated in vitro in a tissue culture environment and their potential to induce protective immunity in sheep Int J Parasitol 31: 359-376.

26. Wikel SK (1999) Tick modulation of host immunity: an important factor in pathogen transmission. Int J Parasitol 29: 851-859.

27. Balic A, Bowles VM, Meeusen EN (2000) The immunobiology of gastrointestinal nematode infections in ruminants. Adv Parasitol 45: 181-241.

28. Willadsen P (2001) The molecular revolution in the development of vaccines against ectoparasites. Vet Parasitol 101: 353-368.
29. Khoo KH, Dell A (2001) Glycoconjugates from parasitic helminths: structure diversity and immunobiological implications. Adv Exp Med Biol 491: 185-205.

30. Nisbet J, Huntley FJ (2006) Progress and opportunities in the development of vaccines against mites, fleas and myiasis causing flies of veterinary importance. Parasite Immunol 28: 165-172.

31. Colebrook E, Wall R (2004) Ectoparasites of livestock in Europe and the Mediterranean region. Vet Parasitol 120: 251-274.

32. Rodríguez M, Rubiera R, Penichet M, Montesinos R, Cremata J, et al. (1994) High level expression of the B. microplus Bm86 antigen in the yeast Pichia pastoris forming highly immunogenic particles for cattle. J Biotechnol 33: 135-146.

33. Williams RB (2002) Anticoccidial Vaccines for broiler chickens: Pathways to success. Avian Pathol 31: 317-353.

34. De Waal DT, Combrink MP (2006) Live vaccines against bovine babesiosis. Vet Parasitol 138: 88-96.

35. Shkap V, Pipano E (2000) Culture-derived parasites in vaccination of cattle against tick-borne diseases. Ann NY Acad Sci 916: 154-171.

36. Buxton D (1993) Toxoplasmosis: the first commercial vaccine. Parasitol Today 9: 335-337.

37. Urquhart GM (1985) Field experience with the bovine lungworm vaccine. Dev Biol Stand 62: 109-112.

38. Marcotty T, Billiouw M, Chaka G, Berkvens D, Losson B, Brandt J (2001) Immunization against East coast by the infection and treatment method: Evaluation of the use of ice baths for field delivery and appraisal of an acid formulation of longacting tetracycline. Vet. Parastiol 99: 175-187.

39. Schetters T, Dubey JP, Adrianarivo A, Frankena K, Romero JJ, et al. (2004) Intervet symposium: bovine neosporosis. Vet Parasitol 125: 137-146.

40. Schijns VE, Tangerås A (2005) Vaccine adjuvant technology: from theoretical mechanisms to practical approaches. Dev Biol (Basel) 121: 127-134.

41. Olson ME, Ceri H, Morck DW (2000) Giardia vaccination. Parasitol Today 16: 213-217.

42. Morrison WI (1989) Immunological control of ticks and tick-borne parasitic diseases of livestock. Parasitology 98 Suppl: S69-86.

43. Taylor MG, Hussein MF, Harrison RA (1990) Baboons, Bovines and Bilherzia vaccines. In Parasitic worms, Zoonioses and Human Health in Africa Unwin Hyman, London pp: 237-259.

44. Boulanger D, Reid CDF, Sturrock RF, Wolowezuk I, Balloul JM, et al. (1991) Immunization of mice and baboons with the recombinant SM28 Glutathione-S-transferase (GST) affects both worm viability and fecundity after experimental infection with Schistosoma mansoni. Parasite immunol 13: 473-490.

45. Haslam SM, Restrepo BI, Obregón-Henao A, Teale JM, Morris HR, et al. (2003) Structural characterization of the N-linked glycans from Taenia solium metacestodes. Mol Biochem Parasitol 126: 103-107.

46. Jarrett WF, Jennings FW, Mcintyre WI, Mulligan W, Urquhart GM (1958) Irradiated helminth larvae in vaccination. Proc R Soc Med 51: 743-744.

47. Ploeger HW (2002) Dictyocaulus viviparus: re-emerging or never been away? Trends Parasitol 18: 329-332.

48. Sharma RL, Bhat TK, Dhar DN (1998) Control of sheep lung worm in India. Parasitol Today 4: 3-36.

49. Miller TA (1971) Vaccination against the canine hookworm diseases. Adv Parasitol 9: 153-183.

50. Lightowlers MW, Colebrook AL, Gauci CG, Gauci SM, Kyngdon CT, et al. (2003) Vaccination against cestode parasites: anti-helminth vaccines that work and why. Vet Parasitol 115: 83-123.

51. Matthews JB, Davidson AJ, Freeman KL, French NP (2001) Immunisation of cattle with recombinant acetylcholinesterase from Dictyocaulus viviparus and with adult worm ES products. Int J Parasitol 31: 307-317.

52. Newton SE, Meeusen EN (2003) Progress and new technologies for developing vaccines against gastrointestinal nematode parasites of sheep. Parasite Immunol 25: 283-296. 
Citation: Kebede B, Sori T, Kumssa B (2016) Review on Current Status of Vaccines against Parasitic Diseases of Animals. J Veterinar Sci Techno 7: 327. doi:10.4172/2157-7579.1000327

Page 8 of 8

53. Schetters T (2005) Vaccination against canine babesiosis. Trends Parasitol 21: 179-184.

54. Nogueira FS, Moreira MA, Borja-Cabrera GP, Santos FN, Menz I, et al. (2005) Leishmune vaccine blocks the transmission of canine visceral leishmaniasis: absence of Leishmania parasites in blood, skin and lymph nodes of vaccinated exposed dogs. Vaccine 23: 4805-4810.
55. Willadsen P (2004) Anti-tick vaccines. Parasitology 129 Suppl: S367-387.

56. Munn EA, Smith TS, Smith H, James FM, Smith FC, et al. (1997) Vaccination against Haemonchus contortus with denatured forms of the protective antigen H11. Parasite Immunol 19: 243-248.

57. Anon (1959) A vaccine against Dictyocaulus viviparous. Vet Rec 71: 153-154. 